\title{
Factors Contributing to Increased Blast Overpressure Inside Modern Ballistic Helmets
}

\author{
Maciej Skotak 1,2,*, Jonathan Salib ${ }^{1}$, Anthony Misistia ${ }^{1}$, Arturo Cardenas ${ }^{1}$, Eren Alay ${ }^{2,3}$, \\ Namas Chandra ${ }^{2}$ and Gary H. Kamimori ${ }^{1, *}$
}

1 Blast Induced Neurotrauma Department, Walter Reed Army Institute of Research, Silver Spring, MD 20910, USA; jonathan.e.salib.ctr@mail.mil (J.S.); anthony.c.misistia.ctr@mail.mil (A.M.); arturo.cardenas@comcast.net (A.C.)

2 Department of Biomedical Engineering, New Jersey Institute of Technology, Newark, NJ 07102, USA; ealay@stevens.edu (E.A.); namas.chandra@njit.edu (N.C.)

3 Department of Computer Science, Stevens Institute of Technology, Hoboken, NJ 07030, USA

* Correspondence: maciej.skotak.ctr@mail.mil (M.S.); gary.h.kamimori.civ@mail.mil (G.H.K.); Tel.: +1-(301)-319-7582 (M.S.); +1-(301)-319-9714 (G.H.K.)

Received: 18 September 2020; Accepted: 9 October 2020; Published: 15 October 2020

check for updates

\begin{abstract}
This study demonstrates the orientation and the "shape factor" have pronounced effects on the development of the localized pressure fields inside of the helmet. We used anatomically accurate headform to evaluate four modern combat helmets under blast loading conditions in the shock tube. The Advanced Combat Helmet $(\mathrm{ACH})$ is used to capture the effect of the orientation on pressure under the helmet. The three modern combat helmets: Enhanced Combat Helmet (ECH), Ops-Core, and Airframe, were tested in frontal orientation to determine the effect of helmet geometry. Using the unhelmeted headform data as a reference, we characterized pressure distribution inside each helmet and identified pressure focal points. The nature of these localized "hot spots" is different than the elevated pressure in the parietal region of the headform under the helmet widely recognized as the under-wash effect also observed in our tests. It is the first experimental study which indicates that the helmet presence increased the pressure experienced by the eyes and the forehead (glabella). Pressure fingerprinting using an array of sensors combined with the application of principle component analysis (PCA) helped elucidate the subtle differences between helmets.
\end{abstract}

Keywords: helmet; blast overpressure; shock wave; peak overpressure; impulse; shock tube

\section{Introduction}

Military service members (SMs) are required to wear combat helmets in training at all times regardless of the type of exercise and weapon system used in order to reduce or eliminate head injuries due to the blunt force trauma. The need for improved ballistic protection led to the development of technologically advanced helmets that outperform their steel-based analogs used in WWI to the Vietnam War era [1]. The invention of high-performance fiber-reinforced polymer-matrix composites resulted in weight reduction, while vastly improving the ballistic protection of modern combat helmets $[1,2]$. The Kevlar-based Advanced Combat Helmet (ACH) has been used since the mid-2000s by combat troops, and more recently, the Enhanced Combat Helmet $(\mathrm{ECH})$ was introduced in 2013. It is made of Dyneema ${ }^{\circledR}$ HB80, an ultra-high-molecular-weight polyethylene (UHMWPE) reinforced with carbon fibers, a thermoplastic that improved ballistic performance and lower density compared to Kevlar [3]. While these helmets were designed for ballistic protection, the recent focus on blast overpressure (BOP) exposure from high-explosives and heavy weapon systems has resulted in questions concerning overpressure inside the helmet [4]. 
To date, research on blast wave propagation inside of the combat helmets is based almost exclusively on data from numerical simulations [5-18]. Advances in medical diagnostic techniques such as magnetic resonance imaging (MRI) and computed tomography (CT) has facilitated the development of high-fidelity, anatomically accurate head models [19-22]. Progress in these fields stimulated the development of high-fidelity computational methods that helped identify mechanisms governing blast loading of the brain without a helmet. The finite element method (FEM) based numerical simulations demonstrated that in the early stages of the blast wave interaction with the head, the intracranial pressure (ICP) is increased (compressive wave) at the coup (impact) side, which is accompanied by the development of the underpressure (tensile wave) on the counter-coup side $[8,18,19,23,24]$. This mechanism is universal and independent of the direction of the blast wave propagation, which was confirmed not only for the front impact but also for the blast waves directed at the head from the side, back, top, or the bottom $[13,15,23]$.

Studies that evaluated the propagation of the blast wave inside of helmets identified the under-wash effect, where the elevated pressure develops under the helmet $[5,6,11,16,25]$ at the back of the head (for frontal impact). A similar mechanism was also confirmed for the back and side incident direction when the $\mathrm{ACH}$ was used in simulations $[14,16,25]$. These reports indicate that the magnitude of the under-wash-induced overpressure on the head exceeds the primary shockwave. However, the correlation between the surface pressure build-up and the corresponding ICP increase is not well researched [5,25].

The blast wave propagation inside combat helmets is a complex problem, with the pressure focusing under the helmet dependent on several factors: (1) the shape of the helmet, (2) orientation relative to the source of the blast wave, and (3) the suspension system (i.e., number of pads, their distribution under the helmet and material properties). The mounting evidence suggests an association between occupational low-level blast exposure and acute adverse neurological effects [26], and related serum biomarkers changes $[27,28]$. The role of the helmet in the development of these effects is unclear at this time. Thus, it is essential that work evaluating the performance of the modern helmet is conducted at relevant blast overpressures (BOPs). Using anatomically accurate headform, we assessed the effect of the four modern combat helmets on the pressure accumulation under the helmet. The scope of work is twofold: (1) evaluate the helmet and blast orientation on pressure under the $\mathrm{ACH}$; and (2) using the $0^{\circ}$ direction to compare under helmet overpressure in the $\mathrm{ECH}, \mathrm{Ops}-\mathrm{Core}$, and Airframe helmets using unhelmeted headform data as a reference.

\section{Materials and Methods}

\subsection{BOP Measurements and Headform Instrumentation}

The details on the blast exposure experiments were published elsewhere [29-31] and briefly described here to familiarize the informed reader with the experimental setup. The shock tube at the New Jersey Institute of Technology (NJIT) is a square cross-section $(0.71 \mathrm{~m} \times 0.71 \mathrm{~m})$ advanced blast simulator with an adjustable breech. The incident BOP is typically monitored along the length of the shock tube using seven high-frequency pressure sensors model 134A24 (PCB Piezotronics, Inc., Depew, NY, USA). In all experiments, the T5 sensor (located in the test section directly above the headform; see Figure 1 in reference [30] for details) was used to measure and quantify the incident overpressure waveforms characteristics. We used circular Mylar membranes with a standard thickness of $0.254(1 \times)$ $\mathrm{mm}$ and $0.726(3 \times) \mathrm{mm}$ stacked together to generate shock waves with two discrete peak overpressures of $70 \mathrm{kPa}$ (approximately $10 \mathrm{psi}$ ) in the test section.

The instrumentation of the customized facial and ocular countermeasures safety (FOCUS) headform [32] was also described in detail in the recently published report [30]. Briefly, we used ten high-frequency piezoelectric sensors, model 102B06, manufactured by PCB Piezotronics (Depew, NY, USA), and mounted flush with the surface of the headform via threaded ports. These sensors are divided into two groups, medial, and circumferential: (1) five medial sensors (marked as H1 to H5, Figure 1B) are located along midline anterior-posterior in $45^{\circ}$ intervals; and (2) five circumferential 
sensors are mounted in the following order: two on the right parietal side ( $\mathrm{H} 6$ and $\mathrm{H} 7$, in $60^{\circ}$ intervals), two in both eye sockets (H8 and H9), and one on the left parietal side of the headform (H10, $90^{\circ}$ interval, Figure 1B). An in-house developed LabView 2016 software was used to capture BOP waveforms. All data were recorded at $1.0 \mathrm{MHz}$ sampling frequency, and the typical acquisition time was $50 \mathrm{~ms}$. The data acquisition system is based on PXI-6133 S Series multifunction DAQ modules and PXIe-1082 PCI Express chassis (National Instruments, Austin, TX, USA). The signals of pressure sensors were fed through 8-channel signal conditioners PCB 483C05 (PCB Piezotronics Inc., Depew, NY, USA) and did not require additional filtration.

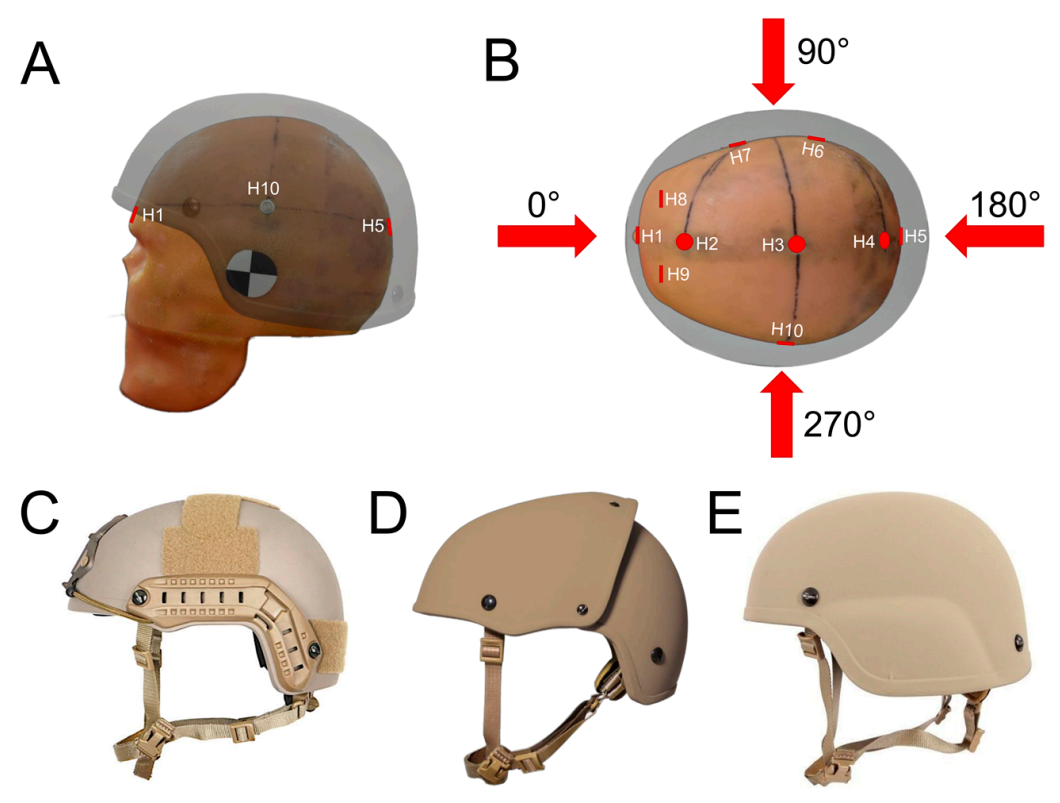

Figure 1. The schematic representation of the headform used in the ballistic helmet performance under blast loading experiments: (A) the headform with the outline of the Advanced Combat Helmet (ACH); (B) the top-view of the headform with the outline of the ACH illustrating the pressure sensor locations (denoted as $\mathrm{H} 1$ to H10) and the direction of the incident shock wave. Three types of helmets used in blast performance tests: (C) Ops-Core, (D) Airframe, and (E) Enhanced Combat Helmet (ECH).

\subsection{Evaluation of Combat Helmets under Blast Overpressure}

The headform was mounted on the Hybrid III neck (Humanetics, Plymouth, MI, USA) [33], in a rigid configuration. The headform-neck assembly was attached to the adapter plate and bolted to the bottom of the shock tube in the test section. The experiments evaluating the performance of various helmets were divided into two groups. The first set of experiments was designed to quantify the effect of orientation on the surface pressure distribution of the headform. The medium size ACH fitted with standard-issue Zorbium ${ }^{\circledR}$ Action Pads (ZAPTM) $6+1$ padding system (Team Wendy, Cleveland, $\mathrm{OH}, \mathrm{USA}$ ) was used for this purpose (Figure 1A). The ACH was mounted onto the headform and secured using a chin strap. The whole assembly was exposed to a single shock wave at two intensity levels, 5 psi and 10 psi nominal shock wave intensity. Four different helmet-headform orientations $\left(0^{\circ}, 90^{\circ}, 180^{\circ}\right.$, and $270^{\circ}$; see Figure $1 \mathrm{~B}$ for the details) were used, and exposures at each combination of orientation-shock wave intensity were repeated six times.

The second set of experiments was designed to evaluate the performance of three modern helmets under blast loading. The following helmets were used: (1) Ops-Core FAST ST high-cut helmet (Gentex, Zeeland, MI, USA; Figure 1C), (2); Airframe ${ }^{\mathrm{TM}}$ high-cut (Crye Precision, Brooklyn, NY, USA; Figure 1D); and (3) ECH (Ceradyne, Inc., Costa Mesa, CA, USA; Figure 1E). The helmets were attached to the headform using the respective chin straps included in the kit, and the same applies to the padding. The headform with the helmet was exposed six times to a shock wave with two nominal intensities: $35 \mathrm{kPa}$ and $70 \mathrm{kPa}$ (approx. $5 \mathrm{psi}$ and $10 \mathrm{psi}$, respectively) at a zero degrees orientation. All helmets used in the tests were brand new with a set of new, unused pads. 


\subsection{Data Reduction and Statistical Analysis}

All waveforms were imported, processed, and quantified in the Origin 2018 software (OriginLab Corp., Northampton, MA, USA). Data from experiments performed at different experimental conditions (shock wave intensity, headform orientation, or helmet type) were pooled together in 3 subsets according to blast intensity. Multiple comparison two-tailed $t$-test with a post-hoc Bonferroni correction was performed, and $p<0.003$ was considered statistically significant. All data are presented as mean and standard deviation.

Principal component analysis (PCA) was performed in RStudio 1.2.5 using R version 3.6.3 [34]. The pressure waveform characteristics (peak overpressure, rise time, duration, and impulse) recorded by the headform sensors were first reduced to non-dimensional amplification factors: their respective values were divided by corresponding incident pressure characteristics. In the next step, the amplification factors were natural log-transformed and subjected to PCA.

\section{Results}

\subsection{The Effect of the Orientation on the Pressure under the Helmet}

In the first part of this research effort, we evaluated the impact of the helmet orientation on the surface pressure distribution under the $\mathrm{ACH}$ (Figure 1B). The representative pressure profiles for the blast exposures performed at $70 \mathrm{kPa}$ incident BOP ( 10 psi) are presented in Figure 2. The highest peak overpressures are noted for the frontal orientation of the headform in the eye sockets $(490 \mathrm{kPa}$, Figure 2A, inset) and on the forehead (H1 sensor, Figure 2A). On the back of the head, the underpressure region develops, as evidenced by the signals from the $\mathrm{H} 4$ and $\mathrm{H} 5$ sensors. The surface pressure in these regions is increased above the incident pressure, while the sensor on the top of the head (H3) and all three circumferential sensors (H6, H7, and H10) report pressure levels similar to the incident pressure.
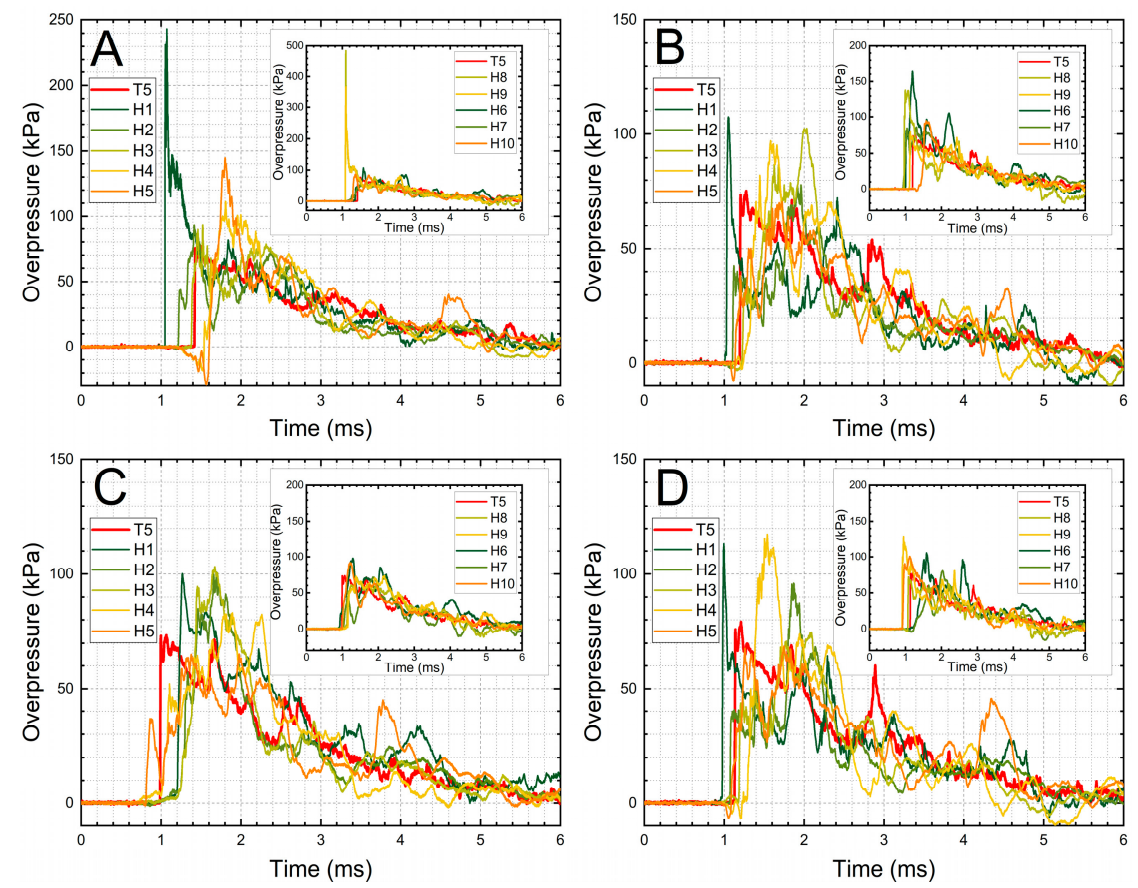

Figure 2. The representative overpressure waveforms recorded at a nominal shock wave intensity of $79 \mathrm{kPa}$ ( $10 \mathrm{psi}$, denoted as T5). The headform equipped with the ACH helmet was oriented at: (A) $0^{\circ}$, (B) $90^{\circ}$, (C) $180^{\circ}$, and (D) $270^{\circ}$ with respect to the direction of the shock wave propagation (see diagram in Figure 1B). The data recorded by the midsagittal (H1-H5) and circumferential (H6-H10, insets) sensors embedded in the headform are presented. 
A very similar pattern is observed for the other three headform orientations. The highest pressures are observed on the side facing the shock wave and on the opposite side. These trends are corroborated when the four characteristics of the pressure waveforms were quantified (Figure 3). The pressure amplification is seen in sensors directly exposed to the incident shock wave ( $\mathrm{H} 1, \mathrm{H} 8$, and $\mathrm{H} 9$ at $0^{\circ} ; \mathrm{H} 1$, $\mathrm{H} 8$, and $\mathrm{H} 6$ at $90^{\circ}$; and $\mathrm{H} 1, \mathrm{H} 9$, and $\mathrm{H} 10$ at $270^{\circ}$ orientation). The same is valid for the sensors under the helmet where the underwash effect is observed (e.g., $\mathrm{H} 4$ and $\mathrm{H} 5$ at $0^{\circ}$ orientation). It is also noteworthy to mention that at $180^{\circ}$, the lowest peak pressures are observed due to shielding of the back face of the helmet preventing the flow of the pressure wave under the helmet. The rise time is a sensitive measure of the shock front dynamics, and at a shorter rise time values, the corresponding loading rates are the highest. The rise time is described often as an "almost instantaneous" increase from ambient to peak pressure. Our results demonstrate that for the incident shock wave with $70 \mathrm{kPa}$ nominal intensity, the rise time is $6 \mu \mathrm{s}$. The rise time is reduced to $3 \mu \mathrm{s}$ at the flat surface facing the blast (H1, Figure 3B). The rise time values increase to $10.5 \mu$ s and $800 \mu$ f for sensors facing away or under the helmet. The variation of the impulse and duration values are less pronounced since these two parameters describe the entire waveform and not only the shock front. Nonetheless, in all four orientations, we noticed amplification above the incident waveform impulse and duration (Figure 3C,D).
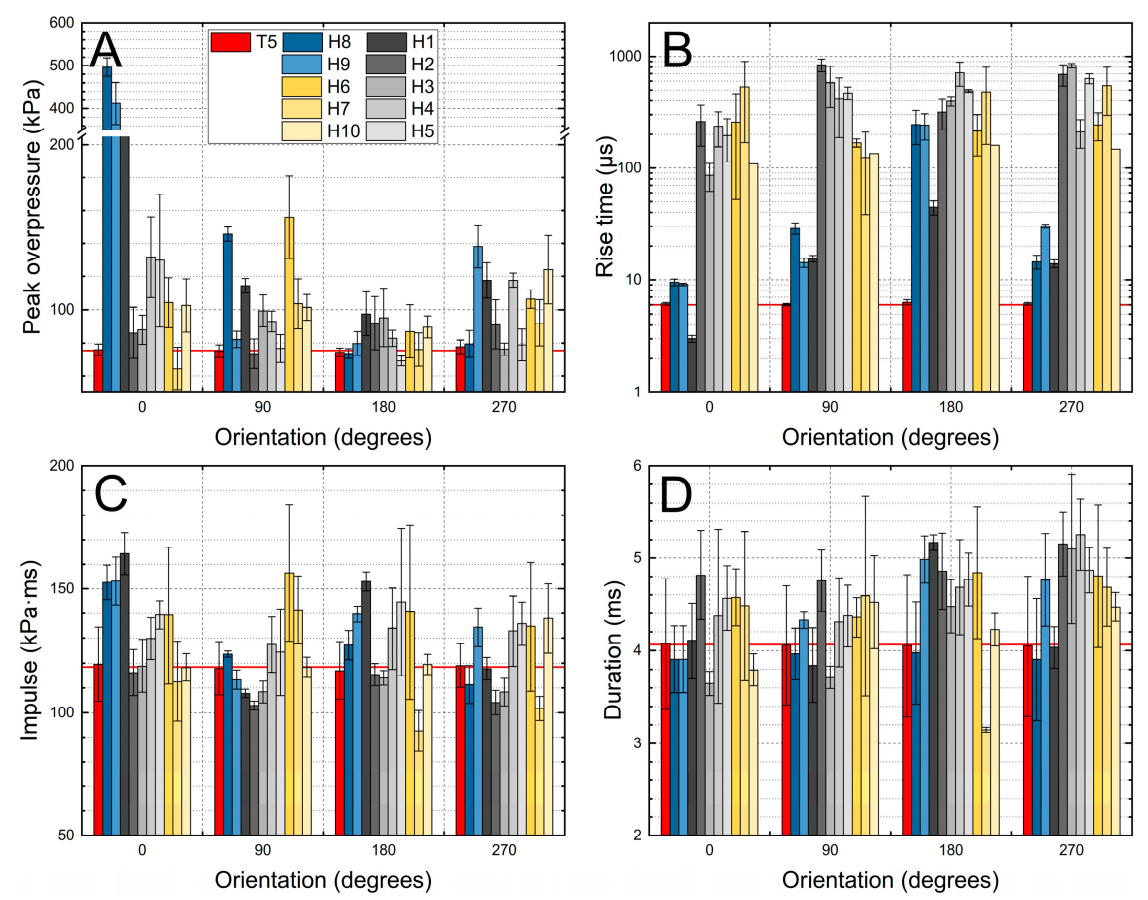

Figure 3. The effect of the orientation on pressure distribution under the helmet. The results of the quantification of the four characteristics of the pressure waveforms: (A) peak overpressure, (B) rise time, (C) impulse, and (D) duration are presented. The data collected at $70 \mathrm{kPa}$ incident blast overpressure (BOP) ( 10 psi, denoted as T5) are expressed as average \pm standard deviation. The red horizontal lines behind the data indicate average incident shock wave characteristics and are plotted as a reference to visualize the trends in the data.

The PCA analysis results are presented in Figure 4. All four characteristics (peak overpressure, rise time, impulse, and duration) for all ten pressure sensors were normalized using their corresponding incident waveform counterparts and natural logarithm normalized. As a result, 40-dimensional space was created for all four headform orientations. The first and second principal components (PC1 and PC2, respectively) describe the variability of $76.3 \%$ of the data. The individual data points for each orientation represent separate shock wave exposures $(n=6)$. The data are clustered according to the headform orientation, and clusters are separated by the PCA algorithm and outlined with $95 \%$ confidence interval ellipses. These results demonstrate that the exposure conditions at $0^{\circ}$ and $180^{\circ}$ 
orientations are the most distinct. The $0^{\circ}$ and $180^{\circ}$ are on the opposite sides of the PC 1 values (i.e., -2.5 to 4.0 and 3.2 to 4.5 , respectively).

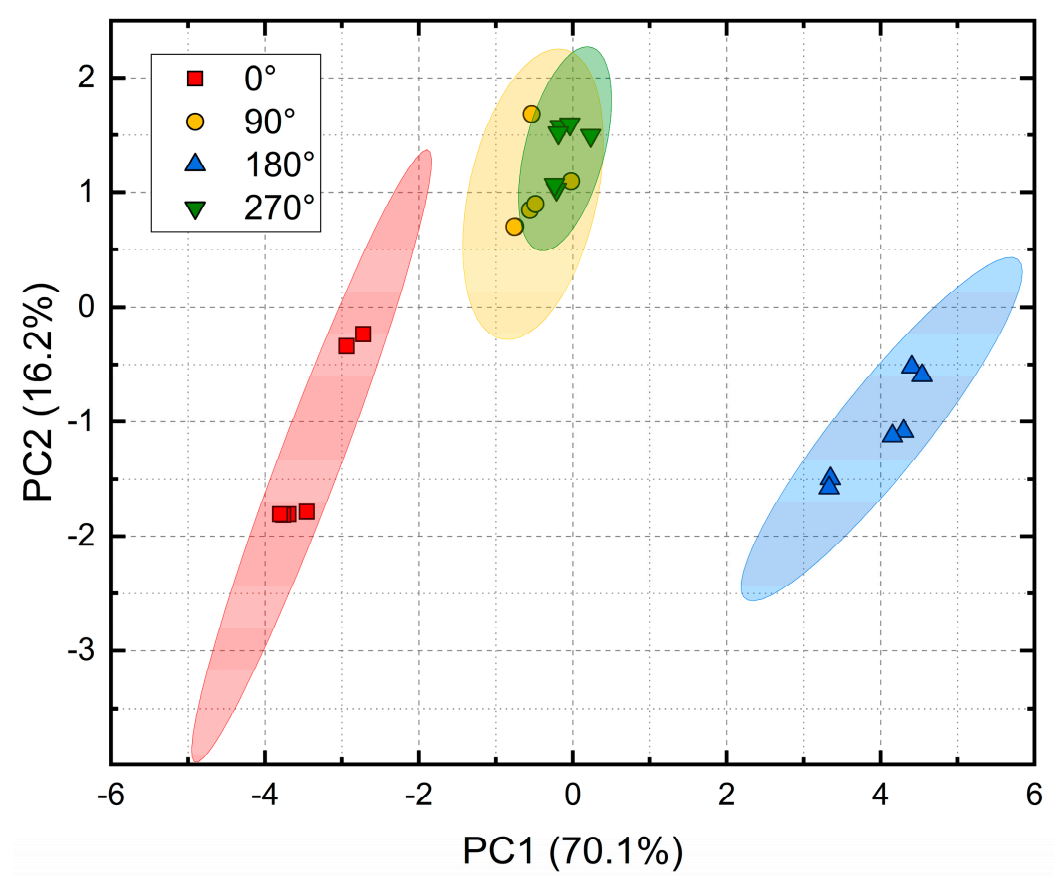

Figure 4. Biplot showing first and second principal components of the surface pressure parameters of the headform equipped with an $\mathrm{ACH}$ helmet. The scores plot illustrates the clustering in the space of two principal components, PC1 and PC2. The data were recorded at $70 \mathrm{kPa}(\sim 10 \mathrm{psi})$ nominal shock wave intensity, and the effect of the headform orientation is presented. The shaded areas surrounding the data points are $95 \%$ confidence ellipses.

On the contrary, the clusters for the $90^{\circ}$ and $270^{\circ}$ orientations data are grouped with significant overlap. It is an expected outcome, considering exposure at both of these orientations are equivalent and observed differences stem from asymmetry in circumferential sensor distribution, i.e., two sensors are located on the right side of the headform (H6 and H7), while only one (H10) was placed on the left side (Figure 2B). Moreover, the aggregate peak overpressure and impulse values for the $90^{\circ}$ and $270^{\circ}$ are very similar (Table 1), unlike the other two orientations tested.

Table 1. The aggregate values (sum of averages) obtained from the quantification of the peak overpressure, rise time, duration, and impulse in the: (1) helmet orientation (ACH), and (2) helmet type (Ops-Core, Airframe, ECH) blast tests. The unprotected headform was used as a reference in the helmet type tests.

\begin{tabular}{ccccc}
\hline & $\begin{array}{c}\text { Peak } \\
\text { Overpressure, kPa }\end{array}$ & Rise Time, ms & Duration, ms & Impulse, kPa·ms \\
\hline Helmet orientation & & & & \\
$0^{\circ}$ & 1856 & 1.70 & 42.2 & 1344 \\
$90^{\circ}$ & 1045 & 2.79 & 42.8 & 1224 \\
$180^{\circ}$ & 842 & 3.30 & 45.1 & 1281 \\
$270^{\circ}$ & 1021 & 3.36 & 47.0 & 1219 \\
Helmet type & & & & \\
Headform & 1610 & 0.17 & 36.3 & 1175 \\
Ops-Core & 1938 & 1.23 & 34.3 & 1226 \\
Airframe & 1910 & 1.90 & 43.7 & 1231 \\
ECH & 2180 & 1.24 & 36.1 & 1297 \\
\hline
\end{tabular}




\subsection{Ops-Core, Airframe and ECH Helmets Blast Testing}

The blast mitigation of the helmets was performed using the headform facing the shock wave $\left(0^{\circ}\right.$ orientation, Figure 1B) at $70 \mathrm{kPa}$ nominal shock wave intensity. The representative pressure traces recorded by sensors mounted flush on the headform surface are presented in Figure 5, and their quantification results are shown in Figure 6. A bare headform was used as a reference, and we evaluated how the geometry of three modern combat helmets affects the surface pressure distribution. The bare headform has the simplest overpressure fingerprint among tested specimens. The highest pressures are observed for the eye sensors (H8 and H9), similar to our previous tests [30,31]. The pressure traces for most sensors resemble the incident overpressure waveform, except for the two sensors located at the front of the head $(\mathrm{H} 1, \mathrm{H} 2)$ and the occipital sensor (H5). Pressure waveforms for all three sensors are more complex (e.g., it would appear the peak overpressure for the $\mathrm{H} 1$ sensor is higher for all helmets ( $240 \mathrm{kPa}$ range, Figure 6A)) compared to bare headform (190 kPa). However, the peak overpressure for the $\mathrm{H} 2$ sensor is reduced under the helmets, from $115 \mathrm{kPa}$ (bare headform) to $70-75 \mathrm{kPa}$ (helmets). The presence of the under-wash effect is evident: the $\mathrm{H} 4$ sensor reports the highest peak overpressures for all three helmets. The $\mathrm{H} 3$ and $\mathrm{H} 5$ sensor peak overpressures are also elevated, exceeding bare headform by $20-40 \mathrm{kPa}$, except for $\mathrm{ECH}$. The rise times are, in general, longer for all sensor locations when helmets are used.
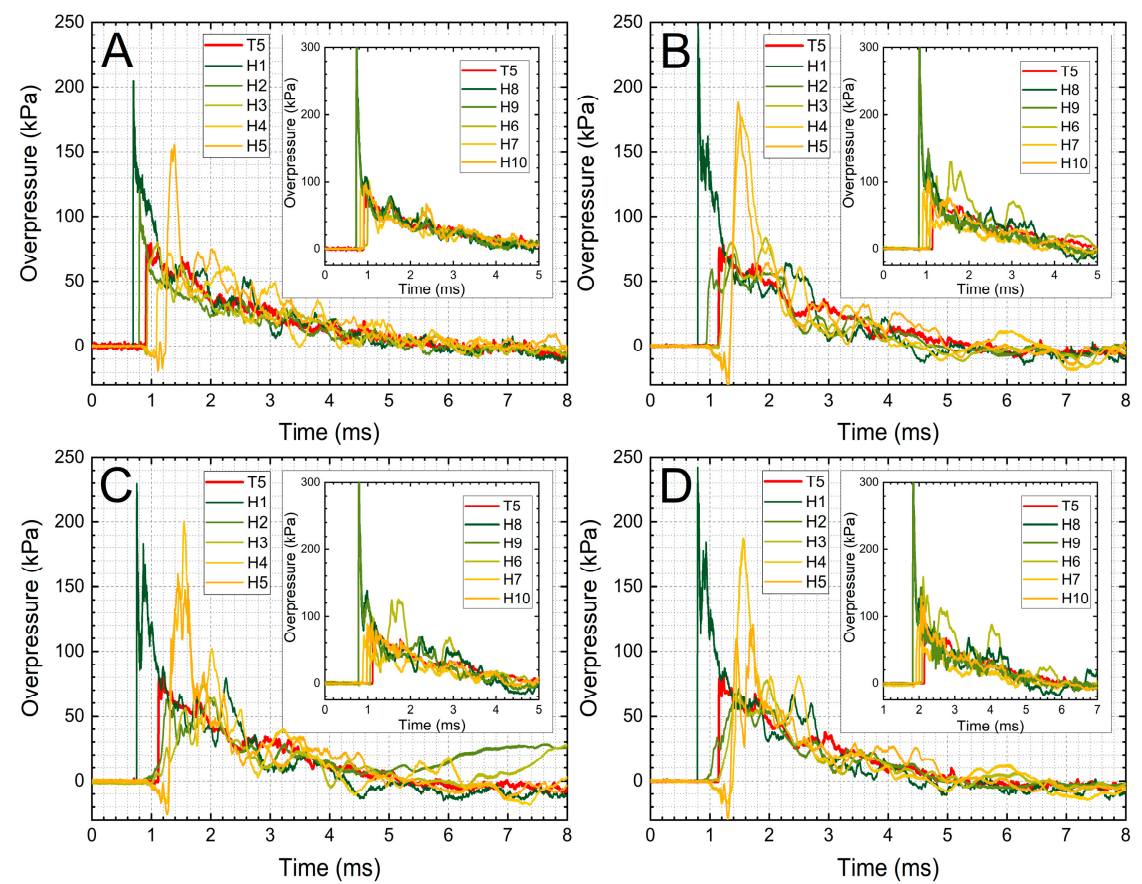

Figure 5. The evaluation of the three helmets under shock wave loading conditions. The representative overpressure waveforms recorded at a nominal shock wave intensity of $10 \mathrm{psi}$ (70 kPa, denoted as T5). The headform with no helmet (A) or equipped with the Ops-Core (B), Airframe (C), or ECH (D) helmets were used in these experiments. The data recorded by the midsagittal (H1-H5) and circumferential (H6-H10, insets) sensors embedded in the headform are presented. 

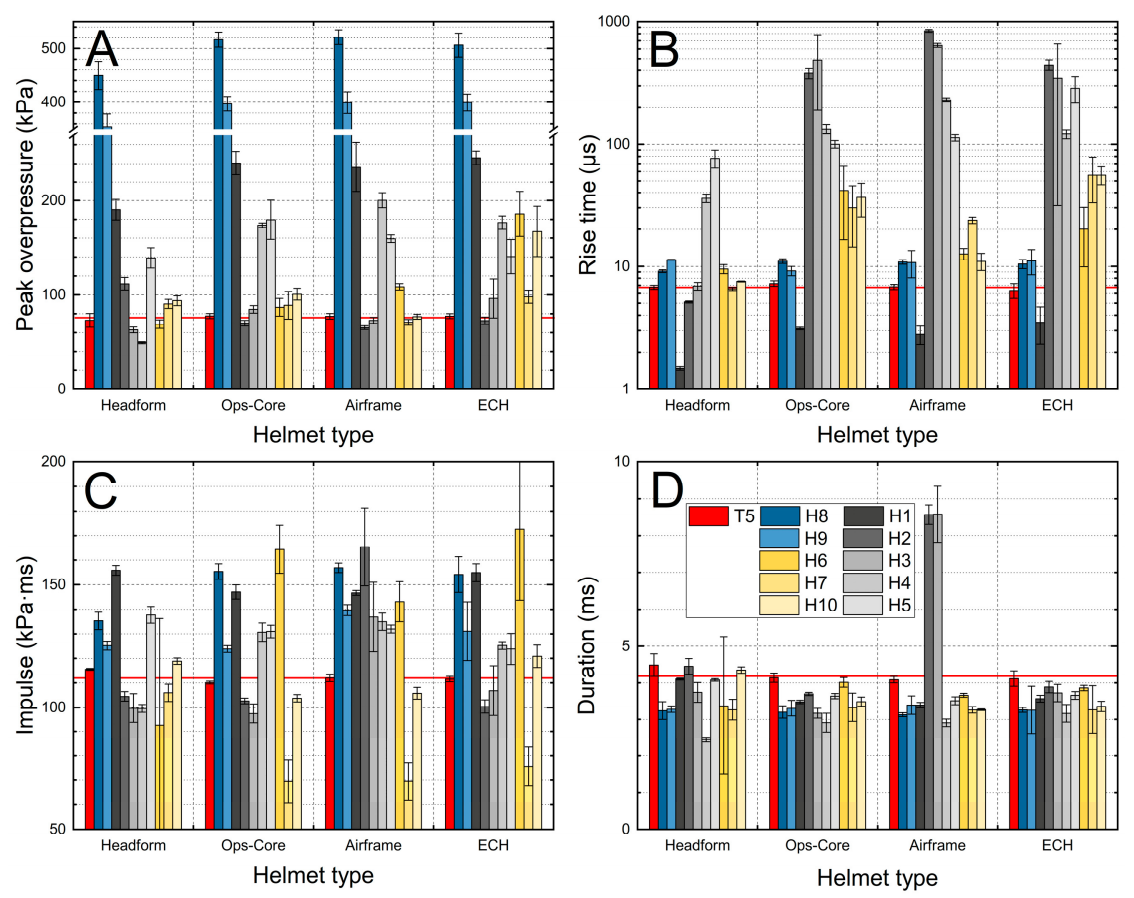

Figure 6. The quantification of peak overpressure (A), rise time (B), impulse (C), and duration (D) recorded for a nominal $\mathrm{BOP}$ of $70 \mathrm{kPa}$. The headform with no helmet (Headform), or equipped with the Ops-Core, Airframe, or ECH helmets were used to measure surface pressure distribution at a nominal shock wave intensity of $70 \mathrm{kPa}$.

Additionally, the $\mathrm{H6}$ sensor located at the temple (Figure 2B) is reporting elevated overpressure exceeding the incident values for Airframe and ECH (Figure 6A). The feature which sets the Airframe helmet apart from the set is secondary pressure pulse at 5-10 ms for the $\mathrm{H} 4$ and $\mathrm{H} 5$ sensors. These signals are not present in the overpressure signatures of the other two helmets and its strong association with the construction of the helmet, and the gap seems extremely likely.

The quantification of the impulse reveals that the H7 sensor reports a decrease of $40 \%$ for the helmets, and this is the only piece of evidence suggesting the protective role of the evaluated helmets. On the contrary, the impulse values for the $\mathrm{H} 6$ sensor are increased by $30-50 \%$ (Figure 6C). The under-wash effect is also evident in this dataset by elevated $\mathrm{H} 4$ impulse values. The Airframe helmet performs the worst in this classification, considering the impulse for the $\mathrm{H} 2$ sensor increases by $60 \%$. The $\mathrm{H} 2$ impulse for the Ops-Core and $\mathrm{ECH}$ remains approximately the same as for the non-helmeted headform.

As a final step of the data analysis, we performed the PCA using the same data reduction algorithm as used in orientation experiments (Figure 7). The two principal components account for $87.6 \%$ of the data variability, which is a very high value. It is evident that the data for the headform are clustered separately and on the opposite side of the PC1 axis compared to all three helmet data clusters. There seems to be a degree of overlap between the ECH and Ops-Core helmets. Simultaneously, the Airframe data cluster is also assigned as a separate entity in the evaluated dataset. The Airframe helmet has the largest aggregate risetime, duration, and impulse values (Table 1). 


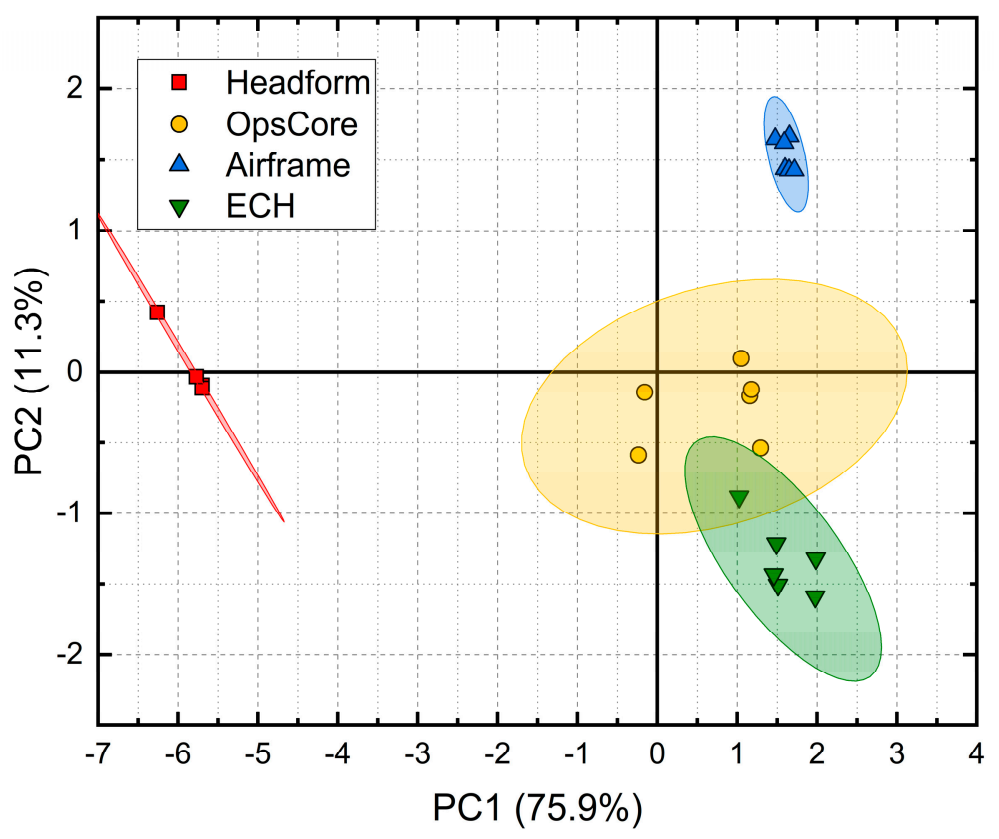

Figure 7. Principal Component Analysis of the surface pressures on the bare headform and equipped with Ops-Core, Airframe, and ECH helmets. The data were recorded at $70 \mathrm{kPa}(\sim 10 \mathrm{psi})$ nominal shock wave intensity, and the scores plot illustrates the clustering in the space of two principal components, PC1 and PC2. The shaded areas surrounding the data points are 95\% confidence ellipses.

\section{Discussion}

In this work, we have evaluated the performance of the four modern combat helmet designs: $\mathrm{ACH}$, $\mathrm{ECH}$, Ops-Core, and Airframe. The ECH is the next generation combat helmet selected a few years ago to replace the $\mathrm{ACH}$. In contrast, the Ops-Core and Airframe are two more recent helmets designed for the Special Operations Community [35]. A literature search summarized in Table 2 indicates a considerable body of work exists on the role of helmets under blast loading using computational approaches. Unlike the theoretical studies, there is a scarcity of published experimental work on the blast mitigation of the helmets $[6,13,18,36]$, and two published only very recently by Kamimori group [37,38]. Most of these studies used ACH in their design, and there are only a few studies that used other helmets (Table 2). The ECH was used in only one study, while no studies evaluated the blast performance of the Ops-Core and Airframe helmets.

The Ops-Core and Airframe are high-cut helmets (Figure 1C,D, respectively), while the ACH and $\mathrm{ECH}$ are low-cut helmets (Figure 1E). Compared to the $\mathrm{ACH} / \mathrm{ECH}$, both high-cut helmets have parts covering the ears on both sides removed. It is a design feature implemented after it was discovered that the water entering ear cups at high speed might pose a safety hazard [39]. The high-cut helmets are designed to incorporate the earmuffs, either as communication devices and/or personal protective equipment (PPE). It seems that the back part of the Ops-Core and Airframe helmets extends further downwards, and they have smaller radii of curvature, and hence a different shape compared to the ECH. A unique feature of the Airframe helmet is a two-shell construction held together by four screws, and a gap between the two parts with the largest separation on the top of the helmet (Figure 1D). The differences in the shape of the helmet (and suspension system) are likely to result in performance variability against blast waves. 
Table 2. The summary of the computational FEM studies which evaluated blast mitigation performance of various combat helmets using high-fidelity human head models. The helmet type, incident peak blast overpressure (reported or calculated) with a corresponding mass of the charge mass (TNT equivalency in $\mathrm{kg}$ ), and standoff distance are presented.

\begin{tabular}{|c|c|c|c|c|}
\hline Helmet Type & $\begin{array}{c}\text { Peak } \\
\text { Overpressure, } \mathrm{kPa}\end{array}$ & $\begin{array}{c}\text { Charge NEW, kg } \\
\text { TNT }\end{array}$ & $\begin{array}{c}\text { Standoff } \\
\text { Distance, m }\end{array}$ & Reference \\
\hline $\mathrm{ACH}$ & $55,230,450^{1}$ & $0.075,0.095$ & $0.75,1.0,2.0$ & [8] \\
\hline $\mathrm{ACH}$ & $260,290^{1}$ & 7.14 & $3.8,4.0$ & [9] \\
\hline $\mathrm{ACH}$ & $450^{2}$ & - & - & [16] \\
\hline PASGT $^{3}$ & 170,326 & 6.85 & $3.0,4.0$ & [17] \\
\hline $\mathrm{ACH}$ & $70,140,200^{4}$ & - & - & [6] \\
\hline $\mathrm{ACH}$ & 520 & 0.07 & 0.58 & {$[23,25]$} \\
\hline $\mathrm{ACH}$ & $407^{1}$ & 0.20 & 1.0 & {$[14]$} \\
\hline PASGT & 103 & 0.0284 & 0.81 & [40] \\
\hline $\mathrm{ECH}$ & 180 & 3.2 & 3.0 & [41] \\
\hline CIPHER $^{5}$ & 120 & 2.1 & 3.0 & [12] \\
\hline $\mathrm{ACH}$ & $270-660$ & $0.85-5.4$ & $1.0-2.8$ & [18] \\
\hline $\mathrm{ACH}$ & $70,150^{4}$ & - & - & [42] \\
\hline $\mathrm{ACH}, \mathrm{LWH}^{6}$ & 1600 & $20^{1}$ & 2.5 & [13] \\
\hline $\mathrm{ACH}$ & 10,000 & 3.16 & 0.12 & [43] \\
\hline $\mathrm{ACH}$ & 520,1886 & $0.07,0.32$ & 0.6 & {$[7]$} \\
\hline $\mathrm{ACH}$ & $195^{1}$ & 2.055 & 3.0 & [11] \\
\hline
\end{tabular}

\subsection{Do Helmets Protect Against the Blast?}

Early work on the effects of the blast waves using computational methods demonstrated that the impulsive loading of the head could lead to tissue level deformation (strain and stress), and by extension, lead to neurological effects $[19,20,45-47]$. As mentioned in the previous section, the effects of helmets on the brain loading were extensively investigated using computational approaches (see Table 2 for details). These studies replicated conditions associated with the detonation of high-explosive materials (e.g., TNT or C4) commonly used in improvised explosive devices (IEDs). Thus, the peak overpressures applied to study the brain response are relatively high, and in the range identified as responsible for mild traumatic brain injury (TBI) in service members during recent conflicts [48].

For an unhelmeted head, previous research demonstrated that on the surface of the coup side, the pressure is amplified significantly, and this amplification is also observed on the counter-coup side $[5,11,19]$. This phenomenon is caused by the blast wave separation as it flows around the head and re-joins at the back, which results in the pressure increase. It was also noted that local geometry is an important parameter in the surface pressure amplification and that anatomical regions with concave geometry like eyes, ears, or nose $[5,12,13]$, experience higher pressure levels as compared to flat surfaces $[13,24]$.

The existing work on the effects of the helmet supports the notion that helmets offer protection at the front of the head (H1 and $\mathrm{H} 2$ locations), but not in the back due to the under-wash effect [5,13,16,25]. Our experimental results are in stark contrast with these findings; there is no evidence that the helmets improve the protection in the forehead, where typically, the highest pressures were observed (Figures $3 \mathrm{~A}$ and 6A). It is possible that the discrepancies in the geometry between experimental and computational models: (1) the gap size between the helmet and the head, and (2) material models used for pad suspension systems could account for these differences. The magnitude of the incident blast wave might also be an important factor: $200 \mathrm{kPa}$ [5], $450 \mathrm{kPa}$ [16], $520 \mathrm{kPa}$ [25], and $1600 \mathrm{kPa}$ [13] incident peak overpressure values were used in these studies, whereas the incident pressure value was merely $70 \mathrm{kPa}$ in our experimental design. Some of these limitations were demonstrated in the published 
work, that attempted validation of numerical models against the experimental data [9]. The decreased volume under the helmet by the unyielding pad suspension system caused the pressure amplification on the surface of the head not covered by pads [12]. Overall, our analysis indicates that all four tested helmets cause surface pressure increases compared to an unprotected headform. The aggregate (sum) peak overpressure for ten pressure sensors is $1610 \mathrm{kPa}$ for the unprotected headform, while for helmets, it is in the range of $1856-2180 \mathrm{kPa}$ (frontal blast loading, Table 2). It appears that each helmet system has a unique surface pressure signature, which was determined by the quantification of surface pressure waveform characteristics followed by PCA (Figures 4 and 7). All helmets increase the pressure in the eyes (H8 and H9) and front of the headform (H1). Further, we noted the pressure increase: (1) on the temple for the ECH (H6 and H10); and (2) on the back (H4 and H5) for the low-cut helmets (Ops-Core and Airframe). The latter had consistently elevated impulse values for all five midsagittal sensors (H1-H5, Figure 6C). There exists some evidence that increase in surface pressure translates into modulation of the ICP [6,25], but this problem was not studied in detail. More work is necessary to provide a definite answer to the question whether pressure amplification on the surface of the head would translate into increase of the ICP and resultant brain injury.

On the contrary, the effects of the blast direction on the brain loading and helmet protection are relatively well understood $[10,13,18,25]$. In these works, the results are presented as pressure histories or peak pressures at a few selected locations on the head surface $[11,13,25]$ where elevated pressures were likely to occur (forehead, back of the head, eyes, and ears) or as peak intracranial pressure $[18,25]$. Previous studies did not attempt to evaluate the surface pressure distribution (head loading) in a collective and integrated way incorporating a larger number of measurement sites (Figures 2 and 6) and blast waveform parameters (Figures 4 and 7), in comparison to the current study. However, even with these limitations, there is a general agreement between these previous studies and results reported herein, i.e., the exposure at the $0^{\circ}$ orientation results in the highest pressure. In contrast, when the blast wave is directed at the back of the head, a significant pressure attenuation was observed. Interestingly, Mott and co-workers noticed that at the $45^{\circ}$ orientation, the pressures reached the highest levels [11].

Considering that the surface pressure changes considerably in response to such factors as helmet shape (design) and blast wave directionality, it is difficult to formulate a simple yes-no answer about helmets' blast protection properties. However, each combination of helmet and blast wave orientation has its own unique "pressure fingerprint", which can be used to identify variables behind the efficacy of helmet design. The surface pressure fingerprinting, dimensionality reduction via PCA and aggregate parameters of the surface pressure (Table 1) combined are necessary to answer the question about the blast mitigation efficacy with a high degree of confidence.

There are several factors that can be considered as limitations of the current work. The padding type was not standardized in our experiments, and the pads we used are a "standard issue suspension system" available with a specific helmet type. The material properties of the pads and their shape and spatial distribution might contribute to the variability of the results. The gap between the headform and the helmet was not controlled and reliant upon the used suspension pad system. The gap size will likely affect the pressure wave flow characteristics and pressure distribution on the headform. The presence of the helmet-mounted performance-enhancing and communication equipment, especially PPE like earmuffs, will likely affect the flow field and pressure distribution under the helmet.

\subsection{Service Applicability Considerations}

As previously mentioned, it must be remembered that the helmet's primary function is to protect the SMs from blunt-force trauma and fragmentation/projectile impacts. Additionally, BOP mitigation has not previously been a consideration when designing and selecting a helmet for Service-wide acceptance. However, there has been a considerable body of research in this area over the last two decades that provides quantifiable data showing the negative outcomes related to an increase in head trauma exacerbated by the current array of issued military helmets $(\mathrm{ACH}, \mathrm{ECH}$, Airframe, and Ops-Core). This research, coupled with others cited herein, have shown that BOP attenuation 
effects caused by helmet geometry to be an imperative consideration when designing and approving a Service helmet for military issuance.

\section{Conclusions}

We demonstrated that the orientation of the incident blast wave results in the heterogeneity of the surface pressure field under the helmet. The frontal orientation consistently demonstrated the highest aggregate peak overpressure, and it was more than two times higher than for the back orientation (presumably due to the contribution of the eye mounted sensors). At the same time, we observed much smaller impulse variability.

This study demonstrated that the shape of the helmet has a pronounced effect on developing the localized overpressure focal points. The nature of these local "hot spots" is different than the widely recognized elevated pressure on the back of the headform ( $\mathrm{H} 4$ and $\mathrm{H} 5$ ) under the helmet shell caused by the under-wash effect (identified in our tests also). It is the first experimental study which indicates that the helmet presence increased the pressure experienced by the eyes (H8 and H9), and the same is also true for the H1 location on the forehead. The "hot spots" were observed on the side of the headform: (1) H6, H7, and H10, corresponding to the temporal bone location for the ECH; and (2) $\mathrm{H} 6$ and $\mathrm{H} 10$ for the Airframe and Ops-Core helmets, respectively. Overall, none of the helmets offer any protection compared to the bare headform, and the Airframe scored the worse thanks to the contribution of extended duration and the highest impulse. Collective pressure measurements at ten distinct locations on the headform indicate that helmets offer no protection against the blast waves. The sum of the peak overpressure for helmeted specimens was, in all cases, higher than for the unprotected headform. The only effect which could be considered as protective, assuming the importance of the loading rates, is the increased rise time for the overpressure waveforms recorded under the helmet.

Author Contributions: Conceptualization, G.H.K., N.C., and M.S.; methodology, G.H.K., J.S., A.M., M.S., and E.A.; validation, J.S., A.C., A.M., and E.A.; formal analysis, M.S.; investigation, J.S., A.M., A.C., E.A., and M.S.; resources, N.C. and G.H.K.; data curation, E.A., J.S., A.M., and A.C.; writing-original draft preparation, M.S. and J.S.; writing-review and editing, M.S. and G.H.K.; visualization, M.S. and J.S.; supervision, G.H.K. and N.C.; project administration, G.H.K. and N.C.; funding acquisition, G.H.K. All authors have read and agreed to the published version of the manuscript.

Funding: This work was supported by an award from the Congressionally Directed Military Research Program (CDMRP), Office of the Assistant Secretary of Defense for Health Affairs, Broad Agency Announcement Award No. W81XWH-16-2-0001 and the Military Operational Medical Research Program (MOMRP), RAD III.

Acknowledgments: Material has been reviewed by the Walter Reed Army Institute of Research. There is no objection to its presentation and/or publication. The opinions or assertions contained herein are the private views of the author, and are not to be construed as official, or as reflecting true views of the Department of the Army or the Department of Defense. The funding received from CDMRP and MOMRP is gratefully acknowledged.

Conflicts of Interest: The authors declare no conflict of interest. The funders had no role in the design of the study; in the collection, analyses, or interpretation of data; in the writing of the manuscript, or in the decision to publish the results.

\section{References}

1. Kulkarni, S.G.; Gao, X.L.; Horner, S.E.; Zheng, J.Q.; David, N.V. Ballistic helmets - their design, materials, and performance against traumatic brain injury. Compos. Struct. 2013, 101, 313-331. [CrossRef]

2. Walsh, S.M.; Scott, B.R.; Spagnuolo, D.M. The Development of a Hybrid Thermoplastic Ballistic Material with Application to Helmets; Army Research Laboratory: Adelphi, MD, USA, 2005.

3. Grujicic, M.; Glomski, P.S.; He, T.; Arakere, G.; Bell, W.C.; Cheeseman, B.A. Material modeling and ballistic-resistance analysis of armor-grade composites reinforced with high-performance fibers. J. Mater. Eng. Perform. 2009, 18, 1169-1182. [CrossRef]

4. United states federal law. In National Defense Authorization Act for Fiscal Year 2020; S.1790-116th Congress: Washington, DC, USA, 2019. 
5. Ganpule, S.; Gu, L.; Alai, A.; Chandra, N. Role of helmet in the mechanics of shock wave propagation under blast loading conditions. Comput. Methods Biomech. Biomed. Eng. 2012, 15, 1233-1244. [CrossRef] [PubMed]

6. Ganpule, S.; Salzar, R.; Perry, B.; Chandra, N. Role of helmets in blast mitigation: Insights from experiments on pmhs surrogate. Int. J. Exp. Comput. Biomech. 2016, 4, 13-31. [CrossRef]

7. Grujicic, M.; Bell, W.C.; Pandurangan, B.; Glomski, P.S. Fluid/structure interaction computational investigation of blast-wave mitigation efficacy of the advanced combat helmet. J. Mater. Eng. Perform. 2010, 20, 877-893. [CrossRef]

8. Hosseini-Farid, M.; Amiri-Tehrani-Zadeh, M.S.; Ramzanpour, M.; Ziejewski, M.; Karami, G. The strain rates in the brain, brainstem, dura, and skull under dynamic loadings. Math. Comput. Appl. 2020, 25, 21. [CrossRef]

9. Li, J.; Ma, T.; Huang, C.; Huang, X.; Kang, Y.; Long, Z.; Liu, M. Protective mechanism of helmet under far-field shock wave. Int. J. Impact Eng. 2020, 103617. [CrossRef]

10. Moss, W.C.; King, M.J.; Blackman, E.G. Skull flexure from blast waves: A mechanism for brain injury with implications for helmet design. Phys. Rev. Lett. 2009, 103, 108702. [CrossRef] [PubMed]

11. Mott, D.R.; Schwer, D.A.; Young, T.; Levine, J.; Dionne, J.-P.; Makris, A.; Hubler, G. Blast-induced pressure fields beneath a military helmet. In Proceedings of the 20th International Symposium on Military Aspects of Blast and Shock, Oslo, Norway, 1-5 September 2008.

12. Mott, D.R.; Young, T.R.; Schwer, D.A. Blast loading on the head under a military helmet: Effect of face shield and mandible protection. In Proceedings of the 52nd Aerospace Sciences Meeting, National Harbor, MD, USA, 13-17 January 2014; p. 0948.

13. Przekwas, A.; Tan, X.G.; Harrand, V.; Reeves, D.; Chen, Z.J.; Sedberry, K.; Chancey, V. Integrated experimental and computational framework for the development and validation of blast wave brain biomechanics and helmet protection. In Proceedings of the HFM-207 NATO Symposium on a Survey of Blast Injury Across the Full Landscape of Military Science, Halifax, NS, Canada, 17 October 2011.

14. Jazi, M.S.; Rezaei, A.; Karami, G.; Azarmi, F. Biomechanical parameters of the brain under blast loads with and without helmets. Int. J. Exp. Comput. Biomech. 2014, 2, 223-244. [CrossRef]

15. Sarvghad-Moghaddam, H.; Rezaei, A.; Eslaminejad, A.; Ziejewski, M.; Karami, G. Mechanical response of the brain under blast: The effect of blast direction and the head protection. In Proceedings of the ASME 2016 International Mechanical Engineering Congress and Exposition, Phoenix, AZ, USA, 11-17 November 2016; Volume 3: Biomedical and Biotechnology Engineering.

16. Sarvghad-Moghaddam, H.; Rezaei, A.; Ziejewski, M.; Karami, G. Cfd modeling of the underwash effect of military helmets as a possible mechanism for blast-induced traumatic brain injury. Comput. Methods Biomech. Biomed. Eng. 2017, 20, 16-26. [CrossRef] [PubMed]

17. Singh, D.; Cronin, D.S. Efficacy of visor and helmet for blast protection assessed using a computational head model. Shock Waves 2017, 27, 905-918. [CrossRef]

18. Zhang, L.; Makwana, R.; Sharma, S. Brain response to primary blast wave using validated finite element models of human head and advanced combat helmet. Front. Neurol. 2013, 4, 88. [CrossRef] [PubMed]

19. Ganpule, S.; Alai, A.; Plougonven, E.; Chandra, N. Mechanics of blast loading on the head models in the study of traumatic brain injury using experimental and computational approaches. Biomech. Modeling Mechanobiol. 2013, 12, 511-531. [CrossRef] [PubMed]

20. Moore, D.F.; Jérusalem, A.; Nyein, M.; Noels, L.; Jaffee, M.S.; Radovitzky, R.A. Computational biology-modeling of primary blast effects on the central nervous system. Neuroimage 2009, 47, T10-T20. [CrossRef]

21. Saunders, R.; Kota, N.; Bagchi, A.; Qidwai, S. On Challenges in Developing a High-Fidelity Model of the Human Head for Traumatic Brain Injury Prediction; AD1063014; Naval Research Lab: Washington, DC, USA, 3 October 2018.

22. Zhang, L.; Yang, K.H.; Dwarampudi, R.; Omori, K.; Li, T.; Chang, K.; Hardy, W.N.; Khalil, T.B.; King, A.I. Recent advances in brain injury research: A new human head model development and validation. Stapp Car Crash J. 2001, 45, 369-394. [PubMed]

23. Sarvghad-Moghaddam, H.; Jazi, M.S.; Rezaei, A.; Karami, G.; Ziejewski, M. Examination of the protective roles of helmet/faceshield and directionality for human head under blast waves. Comput. Methods Biomech. Biomed. Eng. 2015, 18, 1846-1855. [CrossRef]

24. Tan, X.G.; Przekwas, A.J.; Gupta, R.K. Computational modeling of blast wave interaction with a human body and assessment of traumatic brain injury. Shock Waves 2017, 27, 889-904. [CrossRef] 
25. Sarvghad-Moghaddam, H.; Rezaei, A.; Ziejewski, M.; Karami, G. Evaluation of brain tissue responses because of the underwash overpressure of helmet and faceshield under blast loading. Int. J. Numer. Methods Biomed. Eng. 2017, 33, e02782. [CrossRef]

26. LaValle, C.R.; Carr, W.S.; Egnoto, M.J.; Misistia, A.C.; Salib, J.E.; Ramos, A.N.; Kamimori, G.H. Neurocognitive performance deficits related to immediate and acute blast overpressure exposure. Front. Neurol. 2019, 10, 949. [CrossRef]

27. Boutte, A.M.; Thangavelu, B.; LaValle, C.R.; Nemes, J.; Gilsdorf, J.; Shear, D.A.; Kamimori, G.H. Brain-related proteins as serum biomarkers of acute, subconcussive blast overpressure exposure: A cohort study of military personnel. PLoS ONE 2019, 14, e0221036. [CrossRef]

28. Wang, Z.; Wilson, C.M.; Mendelev, N.; Ge, Y.; Galfalvy, H.; Elder, G.; Ahlers, S.; Yarnell, A.M.; LoPresti, M.L.; Kamimori, G.H.; et al. Acute and chronic molecular signatures and associated symptoms of blast exposure in military breachers. J. Neurotrauma 2020, 37, 1221-1232. [CrossRef] [PubMed]

29. Alay, E.; Skotak, M.; Misistia, A.; Chandra, N. Dynamic loads on human and animal surrogates at different test locations in compressed-gas-driven shock tubes. Shock Waves 2018, 28, 51-62. [CrossRef]

30. Skotak, M.; Alay, E.; Zheng, J.Q.; Halls, V.; Chandra, N. Effective testing of personal protective equipment in blast loading conditions in shock tube: Comparison of three different testing locations. PLoS ONE 2018, 13, e0198968. [CrossRef] [PubMed]

31. Skotak, M.; Townsend, M.T.; Alay, E.; Chandra, N. The effect of geometrical factors on the surface pressure distribution on a human phantom model following shock exposure: A computational and experimental study. In Fracture Mechanics Applications; Ozmen, H.B., Balcioglu, H.E., Eds.; IntechOpen: London, UK, 2020.

32. Crowley, J.S.; Brozoski, F.T.; Duma, S.M.; Kennedy, E.A. Development of the facial and ocular countermeasures safety (focus) headform. Aviat. Space Environ. Med. 2009, 80, 831. [CrossRef]

33. Foster, J.K.; Kortge, J.O.; Wolanin, M.J. Hybrid iii-a biomechanically-based crash test dummy. In Proceedings of the 21st Stapp Car Crash Conference, Warrendale, PA, USA, 1 February 1977.

34. R: A Language and Environment for Statistical Computing; $\mathrm{R}$ Development Core Team; R Foundation for Statistical Computing: Vienna, Austria, 2018.

35. South, T. Here's the New Helmet That Socom Operators Will Take into Battle. Available online: https://www.militarytimes.com/news/your-army/2019/04/16/heres-the-new-helmet-that-socom-operatorswill-take-into-battle/ (accessed on 20 June 2020).

36. Ford, M.; Simmonds, K.; Horner, D.; Gauvin, J.; Bagchi, A. Blast response characteristics for an instrumented helmet on a skull-brain surrogate. In Proceedings of the ASME 2011 International Mechanical Engineering Congress and Exposition, IMECE2011, Denver, CO, USA, 11-17 November 2011.

37. Chen, Y.; O’Shaughnessy, T.J.; Kamimori, G.H.; Horner, D.M.; Egnoto, M.J.; Bagchi, A. Role of interfacial conditions on blast overpressure propagation into the brain. Front. Neurol. 2020, 11, 323. [CrossRef] [PubMed]

38. Salib, J.; Egnoto, M.J.; Skotak, M.; LaValle, C.R.; Misistia, A.; Kamimori, G.H. A comparison of the internal and external overpressure for various ballistic helmets. Mil. Med. 2020. under review.

39. Ballistic Military Combat Helmets and Which One Is Right for You! Available online: https://www.hard headveterans.com/blogs/reviews/ballistic-military-helmets-and-which-one-is-right-for-you (accessed on 20 June 2020).

40. Tan, L.B.; Chew, F.S.; Tse, K.M.; Chye Tan, V.B.; Lee, H.P. Impact of complex blast waves on the human head: A computational study. Int. J. Numer. Methods Biomed. Eng. 2014, 30, 1476-1505. [CrossRef]

41. Zhang, T.G.; Satapathy, S.S.; Dagro, A.M.; McKee, P.J. Numerical Study of Head/Helmet Interaction Due to Blast Loading. In Proceedings of the ASME 2013 International Mechanical Engineering Congress and Exposition, San Diego, CA, USA, 15-21 November 2013.

42. Sharma, S.; Makwana, R.; Zhang, L. Evaluation of blast mitigation capability of advanced combat helmet by finite element modeling. In Proceedings of the 12th International LS-DYNA@Users Conference, Dearborn, MI, USA, 3-5 June 2012.

43. Nyein, M.K.; Jason, A.M.; Yu, L.; Pita, C.M.; Joannopoulos, J.D.; Moore, D.F.; Radovitzky, R.A. In silico investigation of intracranial blast mitigation with relevance to military traumatic brain injury. Proc. Natl. Acad. Sci. USA 2010, 107, 20703-20708. [CrossRef]

44. Kingery, C.; Bulmash, G. Air-Blast Parameters from TNT Spherical Air Blast and Hemispherical Surface Blast; ARBRL-TR-02555; Ballistic Research Laboratories: Aberdeen, MA, USA, 1984. 
45. Chafi, M.S.; Karami, G.; Ziejewski, M. Biomechanical assessment of brain dynamic responses due to blast pressure waves. Ann. Biomed. Eng. 2010, 38, 490-504. [CrossRef]

46. Roberts, J.C.; Harrigan, T.P.; Ward, E.E.; Taylor, T.M.; Annett, M.S.; Merkle, A.C. Human head-neck computational model for assessing blast injury. J. Biomech. 2012, 45, 2899-2906. [CrossRef]

47. Taylor, P.A.; Ford, C.C. Simulation of blast-induced early-time intracranial wave physics leading to traumatic brain injury. J. Biomech. Eng. 2009, 131, 061007. [CrossRef] [PubMed]

48. Chandra, N.; Sundaramurthy, A.; Gupta, R.K. Validation of laboratory animal and surrogate human models in primary blast injury studies. Mil. Med. 2017, 182, 105-113. [CrossRef] [PubMed]

Publisher's Note: MDPI stays neutral with regard to jurisdictional claims in published maps and institutional affiliations.

(C) 2020 by the authors. Licensee MDPI, Basel, Switzerland. This article is an open access article distributed under the terms and conditions of the Creative Commons Attribution (CC BY) license (http://creativecommons.org/licenses/by/4.0/). 